\title{
RINGS WHOSE MODULES ARE PROJECTIVE OVER ENDOMORPHISM RINGS
}

\author{
ROBERT L. SNIDER
}

ABSTRACT. Let $R$ be either an Artin ring or a commutative ring. We show that every $R$-module is projective over its endomorphism ring if and only if $R$ is uniserial.

We study rings, all of whose modules are projective over their endomorphism rings. We show that for Artin or commutative rings this is equivalent to being uniserial. We remark that we know of no examples other than uniserial rings and conjecture that all such rings are uniserial. Our proof uses the complete ring of quotients as in [4].

Our work is partially motivated by the work of Sally and Vasconcelos on commutative rings whose ideals are projective over their endomorphism rings [7].

We use $J$ throughout to denote the Jacobson radical and Soc $(R)$ to denote the socle of $R$.

\section{Artin $U$-rings.}

Definition. A ring $R$ is a left $U$-ring if every left $R$-module is projective over its endomorphism ring.

Proposition 1. If $S$ is Morita equivalent to a left $U \cdot r i n g ~ R$, then $S$ is a left U-ring.

Proof. There exists a finitely generated projective generator $M_{R}$ such that $S=\operatorname{End}_{R}(M)$. If $B$ is an $S$-module, then there exists an $R$-module $A$ with $M \otimes_{R} A \cong B$. Now $\operatorname{End}_{R}(A) \cong \operatorname{End}_{S}(B)$ by $f \rightarrow 1 \otimes f$. Also there exists a right $R$-module $N$ with $M \oplus N=R^{n}$. Hence

$$
\left(M \otimes_{R} A\right) \oplus\left(N \otimes_{R} A\right) \cong(M \oplus N) \otimes_{R} A \cong R^{n} \otimes_{R} A \cong A^{n} .
$$

Note all these isomorphisms are right $\operatorname{End}_{R}(A)$-isomorphisms. Therefore $B \cong M \otimes_{R} A$ is projective over $\operatorname{End}_{S}(B)$ since $A^{n}$ is projective over $\operatorname{End}_{R}(A)$.

Received by the editors July 25, 1973. AMS (MOS) subject classifications (1970). Primary 16A36. Key words and phrases. Uniserial ring, projective module, endomorphism ring. 
Lemma 2. If $R$ is a left Artin U-ring and e a primitive idempotent, then $\mathrm{Re}$ contains a submodule isomorphic to $\mathrm{Re} / \mathrm{J} e$.

Proof. $R=R e_{1} \oplus \cdots \oplus R e_{n}$ where the $e_{i}$ 's are primitive idempotents and $e=e_{1}$.

Case 1. $R e_{1} \neq R e$ for $i>1$. If $e_{i} R e_{1}=0$ for $i>1$, then the lemma is proved since $\operatorname{Soc}\left(R e_{1}\right) \neq 0$ and $\operatorname{Hom}\left(R e_{i}, R e_{1}\right)=e_{i} R e_{1}=0$ and hence the only possible simple submodules of $R e_{1}$ are $R e_{1} / J e_{1}$.

Suppose then that $e_{2} R e_{1} \neq 0$. Let $S=R e_{1} / J e_{1}$ and $M=R e_{1} \oplus S$. Suppose further that $R e_{1}$ contains no copy of $S$.

$$
\operatorname{End}(M)=\left(\begin{array}{ll}
\operatorname{Hom}\left(R e_{1}, R e_{1}\right) & \operatorname{Hom}\left(R e_{1}, S\right) \\
\operatorname{Hom}\left(S, R e_{1}\right) & \operatorname{Hom}(S, S)
\end{array}\right) .
$$

$\operatorname{Hom}\left(R e_{1}, R e_{1}\right)=e_{1} R e_{1} \cdot \operatorname{Hom}\left(R e_{1}, S\right)=e_{1} R e_{1} / e_{1} J e_{1}$ and $\operatorname{Hom}(S, S)$ $=e_{1} R e_{1} / e_{1} J e_{1}$. Therefore

$$
\operatorname{End}(M)=\left(\begin{array}{cc}
e_{1} R e_{1} & e_{1} R e_{1} / e_{1} J e_{1} \\
0 & e_{1} R e_{1} / e_{1} J e_{1}
\end{array}\right)
$$

Now $R e_{1}=e_{1} R e_{1} \oplus\left(1-e_{1}\right) R e$ whence

$$
M=R e_{1} \oplus S=\left(e_{1} R e_{1} \oplus S\right) \oplus\left(\left(1-e_{1}\right) R e_{1} \oplus 0\right) .
$$

Since $R e_{1} \neq R e_{i}$ for $i>1,\left(1-e_{1}\right) R e_{1} \subseteq J$. Therefore the above decomposition is as $\operatorname{End}(M)$ modules whence $\left(1-e_{1}\right) R e_{i} \oplus 0$ is a projective End $(M)$ module. Also $\left(1-e_{1}\right) R e_{1}$ is projective over the local ring $e_{1} R e_{1}$ $=\operatorname{End}\left(R e_{1}\right)$. Therefore $\left(1-e_{1}\right) R e_{1} \cong \bigoplus \Sigma e_{1} R e_{1}$ as an $e_{1} R e_{1}$-module and hence as an $\operatorname{End}(M)$ module. Therefore

$$
e_{1} R e_{1} \cong\left(\begin{array}{cc}
e_{1} R e_{1} & e_{1} R e_{1} / e_{1} J e_{1} \\
0 & e_{1} R e_{1} / e_{1} J e_{1}
\end{array}\right) /\left(\begin{array}{cc}
0 & e_{1} R e_{1} / e_{1} J e_{1} \\
0 & e_{1} R e_{1} / e_{1} J e_{1}
\end{array}\right)
$$

But

$$
\left(\begin{array}{cc}
0 & e_{1} R e_{1} / e_{1} J e_{1} \\
0 & e_{1} R e_{1} / e_{1} J e_{1}
\end{array}\right)
$$

is not a right ideal summand of $\operatorname{End}(M)$ and hence $e_{1} R e_{1}$ is not projective, a contradiction.

Case 2. $R e_{1} \cong R e_{i}$ for some $i>1$. Let $M=R e_{1} \oplus R e_{i 1} \oplus \cdots \oplus R e_{i r}$ 
where $R e_{i} \neq R e_{i j}$ and each $R e_{j} \cong R e_{i k}$ for some $k . M$ is a progenerator and $S=\operatorname{End}_{R}(M)$ is Morita equivalent to $R$. Then $S=S f_{1} \oplus \cdots \oplus S f_{r}$ where $S f_{i} \neq S f_{j}$ for $i \neq j$. By Case 1, each indecomposable projective $S f$ contains $S f / J f$. The Morita equival ence of $R$ and $S$ now easily gives the result for $R$.

Proposition 3. If $R$ is a left Artin U-ring, then $R$ is uniserial.

Proof. As in the proof of the lemma, replace $R$ by a Morita equivalent ring $S$ where $S=S e_{1} \oplus \cdot \cdots \oplus S e_{n}$ where the $e_{i}$ 's are primitive idempotents and $S e_{i} \neq S e_{j}$ for $i \neq j$. Each maximal left ideal of $S$ is a two-sided ideal since $S / J$ is a direct sum of fields. Since each type of simple module is contained in $S$, we see that the right annihilator of $I$ is nonzero for each maximal ideal $I$. Therefore no proper left ideal of $S$ is dense [4, p. 96]. Hence, it follows that $S$ is its own complete ring of quotients. Let $E$ be the injective hull of $S$ and $H=$ End $_{S} E$. Since $S$ is its own quotient ring, $S=\operatorname{End}_{H}(E)$.

$S$ has exactly $n$ isomorphism classes of simple modules. Let $M_{1}, \cdots$, $M_{n}$ be nonisomorphic simples. Now

Hence

$$
\operatorname{Soc}(S)=\sum_{i=1}^{n} \sum_{j=1}^{t_{i}} M_{i j} \text { where } M_{i j} \cong M_{i} .
$$

$$
E=\sum_{i=1}^{n} \sum_{i=1}^{t_{i}} E\left(M_{i j}\right) .
$$

$H$ is semiperfect by [4, Proposition 2, p. 103]. Also 1 in $H$ is the sum of $m=\Sigma_{i=1}^{n} t_{i}$ projections. These projections are primitive idempotents. If $\pi_{i j}$ is the projection of $E$ onto $E\left(M_{i j}\right)$, then $\pi_{i j} H \cong \pi_{i n} H$ since $E\left(M_{i j}\right) \cong$ $E\left(M_{i n}\right)$. Therefore, $H$ has at most $n$ isomorphism classes of indecomposable projectives. $E$ is a sum of $n$ indecomposable $H$ modules since the $e_{i}$ 's are primitive orthogonal idempotents and $S=\operatorname{End}_{H}(E)$. Also these indecomposable $H$ summands are not isomorphic since $S e_{i} \neq S e_{j}$ for $i \neq j . E$ is generated by 1 as an $H$ module.

Therefore $E$ contains all types of finitely generated indecomposable $H$-projectives as $H$-summands and hence $E$ is a finitely generated projective $H$ generator. By the theory of Morita equivalence, $E$ is a finitely generated projective generator over $\operatorname{End}_{H}(E)=S$. It now follows easily that $S$ is selfinjective and hence quasi-Frobenius. Therefore $R$ is quasi-Frobenius. Since each homomorphic image of a $U$-ring is a $U$-ring, each image of $R$ is $Q F$ and hence by a theorem of Nakayama [S], $R$ is uniserial. 
Proposition 4. If $R$ is uniserial, then $R$ is a U-ring.

Proof. It clearly suffices to assume $R$ is indecomposable and local. Let $M$ be an $R$-module. $M=\bigoplus \Sigma_{I} N_{i}$. Clearly, we may assume $I$ is well ordered and $l\left(N_{i}\right) \geq l\left(N_{j}\right)$ if $i \leq j .\left(l(M)\right.$ is the length of $\left.M_{\text {. }}\right)$ If $l\left(N_{i}\right) \leq l\left(N_{s}\right)$, then $\operatorname{Hom}\left(N_{i}, N_{s}\right) \cong N_{i}$ and $\operatorname{Hom}\left(N_{S}, N_{i}\right) \cong N_{i}$. End $(M)$ is an infinite "matrix" ring $\operatorname{Hom}\left(N_{i}, N_{j}\right)$. We see that $M$ is isomorphic to the first row.

Combining Propositions 3 and 4, we obtain

Theorem 5. An Artin ring is a U-ring if and only if it is uniserial.

We note that Faith has proven that if $R$ is a ring for which every $R$ module $M$ is finitely generated and projective over $\operatorname{End}(M)$ and also $R / \operatorname{Ann}(M)$ $\cong \operatorname{End}_{\mathrm{End}(M)}(M)$, then $R$ is uniserial [2]. This is an immediate corollary of our result since balanced rings are Artin [1].

\section{Commutative $U$-rings.}

Lemma 1. If $R$ is a commutative U-ring and $S$ is a multiplicatively closed set, then $S^{-1} R$ is a U-ring.

Proof. If $M$ is an $S^{-1} R$ module, then $\operatorname{End}_{R}(M)=\operatorname{End}_{S^{-1}}(M)$.

Lemma 2. A local domain $R$ is a U-ring if and only if $R$ is a field.

Proof. Let $M$ be the maximal ideal of $R$ and $E$ the injective hull of $R / M$. End $(E)$ is a torsion free $R$-module. If $E$ were a projective End $(E)$ module, then $E \subseteq \bigoplus \Sigma_{I}$ End $(E)$, and hence $E$ would be torsion free, a contradiction.

Lemma 3. A commutative von Neumann regular U-ring is semisimple Artin.

Proof. Let $R$ be regular and $Q$ the complete ring of quotients of $R$. Let $I$ be an essential ideal of $Q$. End $Q(I)=Q$ since $Q$ is self-injective and $\operatorname{Ann}(I)=0$. Let $f$ be in $\operatorname{End}_{R}(I)$. $f$ extends to $\bar{f}$ in $Q$ since $Q$ is the injective hull of $R$ and $Q=\operatorname{End}_{R}(Q)$. The map $f \rightarrow \bar{f}$ is an isomorphism. Therefore $I$ is a projective $Q$-module since $R$ is a $U$-ring and hence $Q$ is hereditary. A regular hereditary self-injective ring is semisimple Artin by a theorem of Osofsky [6]. It follows that $Q$, and hence $R$, has only finitely many idempotents whence $R$ is semisimple Artin.

Theorem 4. A commutative ring is a U-ring if and only if it is uniserial.

Proof. Uniserial rings are $U$-rings by Theorem 5 above. Conversely, 
let $R$ be a commutative $U$-ring. We first show $R$ has Krull dimension 0 . Suppose then that $P$ is a nonmaximal prime ideal and $M$ is a maximal ideal containing $P . R / P$ is a $U$-ring and hence by Lemma $1, R / P$ localized at $M / P$ is a $U$-ring. This contradicts Lemma 2 and hence $R$ has Krull dimension 0 . If $N$ is the nilradical, then $R / N$ is von Neumann regular [4] and hence semisimple Artin by Lemma 3. Since idempotents may be lifted mod $N$, $R$ is a finite direct sum of local rings. We may clearly assume $R$ itself is local with nil maximal ideal $N$. We may also assume $N \neq 0$. Let $x$ in $N$, $x \neq 0$, and choose an ideal $I$ maximal with respect to $x$ not in $I . R / I$ is subdirectly irreducible. Since $R / I$ is local with a unique minimal ideal, $R / I$ is its own complete ring of quotients. As in the proof of Proposition 3 of $\S 1, R / I$ is self-injective. It follows that every endomorphism of $N / I$ is given by multiplication and hence $\operatorname{End}(N / I) \cong(R / I) / \operatorname{Ann}(N / I)$. $N / I$ is indecomposable over this local ring and hence must be isomorphic to End $(N / I)$ whence $N / I$ is cyclic. Since $N$ is nil and $N / I$ is cyclic, there exists an integer $r$ with $N^{r} \subseteq I$. Therefore $N^{2} \neq N$. Since every Artin homomorphic image of $R / N^{2}$ is uniserial, by Theorem 5 above, it follows that $R / N^{2}$ is uniserial and by induction $R / N^{s}$ for all positive integers $s$. Let $x$ be an element of $N$ with $x$ not in $N^{2}$. Then $x$ generates $R / N^{s}$. Since $x$ is nilpotent it follows that $N^{s}=0$ for some $s$ and hence $R$ is uniserial.

\section{REFERENCES}

1. V. Dlab and C. M. Ringel, Balanced rings, Lectures on Rings and Modules, Lecture Notes in Math., vol. 246, Springer-Verlag, New York, 1972.

2. C. Faith, Modules finite over endomorphism ring, Lectures on Rings and Modules, Lecture Notes in Math., vol. 246, Springer-Verlag, New York, 1972.

3. I. Kaplansky, Algebraic and analytic aspects of operator algebras, Amer. Math. Soc., Providence, R. I., 1970. $34 \# 5857$.

4. J. Lambek, Lectures on rings and modules, Blaisdell, Waltham, Mass., 1966.

5. T. Nakayama, On Frobeniusean algebras. I, Ann. of Math. 40 (1939), 611-633. MR 1, 3.

6. B. Osof sky, Rings all of whose finitely generated modules are injective, Pacific J. Math. 14 (1964), 645-650. MR 28 \#5090.

7. J. Sally and W. Vasconcelos, Stable rings (to appear).

DEPARTMENT OF MATHEMATICS, NORTHWESTERN UNIVERSITY, EVANSTON, ILLINOIS 60201

Current address: Department of Mathematics, Virginia Polytechnic Institute and State University, Blacksburg, Virginia 24061 